\title{
Flame Surface Properties of Premixed Flames in Isotropic Turbulence: Measurements and Numerical Simulations
}

\author{
S. KWON, M.-S. WU, J. F. DRISCOLL, and G. M. FAETH* \\ Department of Aerospace Engineering, The University of Michigan, Ann Arbor, MI 48109-2140
}

\begin{abstract}
An experimental and theoretical investigation of free turbulent premixed flames propagating in isotropic turbulence at neutrally stable preferential diffusion conditions is described. Experiments were limited to the wrinkled thin laminar flamelet regime and involved mixtures of hydrogen, air, and nitrogen ignited within a fan-stirred combustion chamber. Measurements included flame tomography for flame surface statistics and two-point laser velocimetry for unburned gas turbulence properties. Flame surface properties were numerically simulated using a two-dimensional flame propagation algorithm combined with statistical time series simulation of unburned gas velocities along the flame surface. Measurements showed progressively increasing flame radius fluctuations, flame surface fractal dimensions, and turbulent/laminar flame perimeters with increasing mean frame radius. The rate of increase of these properties all increased with increasing turbulence intensities relative to the laminar flame speed. Simulated flame properties duplicated these trends but underestimated the effects of turbulence-a deficiency mainly attributed to the limitations of a two-dimensional simulation. Extension of the method to a three-dimensional simulation, to obtain a more definitive evaluation of the simulation, appears to be computationally feasible.
\end{abstract}

\section{NOMENCLATURE}

$A_{L} \quad$ area of mean flame surface.

$A_{T}$

$D_{3}$

$\mathrm{D}_{2}$

$E_{i}(f)$

$f$

$f(\Delta x)$

$F$

$g(\Delta x)$

G

$l_{K}$

$L$

$N$

$\mathrm{O}_{2} /\left(\mathrm{N}_{2}+\mathrm{O}_{2}\right)$

$P_{L}$ average surface area of wrinkled flame surface

fractal dimension of flame surface

fractal dimension of intersection of flame surface with a plane temporal power spectral density of velocity component $i$

frequency

longitudinal spatial correlation coefficient

value of $f(\Delta x)=f(\Delta y)$

transverse spatial correlation coefficient

value of $g(\Delta x)=g(\Delta y)$

Kolmogorov length scale

average flame surface perimeter

for ruler of length $\epsilon$

fan speed

volumetric fraction of $\mathrm{O}_{2}$ in nonfuel gas

perimeter of mean flame surface

${ }^{*}$ Corresponding author.

$P_{T}$

$r$

$r_{f}$

$\mathrm{Re}_{T}$

$s_{u 0}, s_{y 0}$

$S_{L}, S_{T}$

$t$

$r$

$u, v$

$U_{0 i}, V_{0 i}$

$x, y$

\section{Greek Symbols}

$\alpha$
$\delta_{\mathrm{L}}$
$\Delta t$
$\Delta x, \Delta y$
$\Lambda, \Lambda_{\mathrm{f}}$
$\Lambda_{g}$

$\alpha$

$\Delta t$

$\Lambda, \Lambda$ average perimeter of wrinkled flame surface

radial distance

flame radius

Reynolds number of turbulence, $\bar{u}^{\prime} \Lambda / \nu$

uncorrelated Gaussian random shock for $u$ and $v$ velocity components at point 0

laminar and turbulent burning velocity

time

value of temporal correlation coefficient at $\Delta t$

vertical (radial) and horizontal velocities

weighting factors in autoregressive process

orthogonal coordinate directions thermal diffusivity

laminar flame thickness

time increment

orthogonal distance increments

longitudinal integral length scale transverse integral length scale for exponential approximation 


$\begin{array}{ll}\nu & \text { kinematic viscosity } \\ \rho & \text { density } \\ \tau & \text { integral time scale } \\ \phi & \text { fuel-equivalence ratio }\end{array}$

\section{Subscripts}

$\begin{array}{ll}b & \text { burned gas } \\ u & \text { unburned gas }\end{array}$

\section{Superscripts}

$$
\left(\overline{)},()^{\prime}\right.
$$

time-averaged mean and rms fluctuating property

\section{INTRODUCTION}

An experimental and theoretical investigation of free turbulent premixed flames propagating in isotropic turbulence at neutrally stable preferential diffusion conditions is described. This problem is of interest because the constraints of flame holding and the complications of effects of preferential diffusion are absent, while the flame is subjected to the simplest hydrodynamic state of turbulence. The experiments involved mixtures of hydrogen, air, and nitrogen ignited within a fanstirred combustion chamber. Measurements included frame tomography to find flame surface statistics and two-point laser velocimetry to find the turbulence properties of the unburned gas, similar to recent work on turbulent premixed jet flames in this laboratory [1]. Test conditions yielded turbulent Reynolds numbers of $0-4195$ and turbulence intensities relative to the laminar flame speed in the range $0-1.6$, with $l_{L} / \delta_{K}$ on the order of 10 . Thus, the experiments were in the wrinkled thin laminar flamelet regime with turbulence levels typical of practical applications $[2,3]$. Flame surface properties were numerically simulated using a flame propagation algorithm coupled with statistical time series simulation [4] of unburned gas velocities along the flame surface. This approach was examined because it offers a computationally tractable treatment of flame surface distortion by turbulence.

Within the thin flamelet regime, preferential diffusion involves the interaction between a more rapidly diffusing reactant and the variation of laminar burning velocity such that increasing or decreasing laminar burning velocity with increas- ing concentration of the more rapidly diffusing reactant yields unstable or stable flames [5]. Hydrogen is the more rapidly diffusing reactant in hydrogen/air/nitrogen mixtures, which have a maximum laminar flame speed at a fuel-equivalence ratio of $1.8[6,7]$; therefore, these flames are unstable or stable for fuel-equivalence ratios below or above this fuel-equivalence ratio. Recent work in this laboratory showed that effects of preferential diffusion instability are important for turbulent premixed hydrogen/air flames with turbulence distortion of the flame surface enhanced for unstable conditions and retarded for stable conditions $[1,8]$. Thus, present experiments were carried out at $\phi=1.8$, where preferential diffusion effects are suppressed.

Other forms of instabilities, like RayleighTaylor and hydrodynamic instabilities, also were not important for present conditions. RayleighTaylor instabilities due to effects of buoyancy were not significant because flame velocities were relatively high, ca. $10 \mathrm{~m} / \mathrm{s}$, similar to earlier work on free turbulent flames [9-17]. Additionally, hydrodynamic instabilities appear to be weak and only have been observed for rather large diameter laminar flames; see Groff [11] and references cited therein. Finally, laminar flame tests in still gases gave no evidence for either of these instabilities over the present range.

Earlier measurements of free turbulent premixed flames in isotropic turbulence are reported by Abdel-Gayed and Bradley [9], Abdel-Gayed et al. [10], Groff [11], Mantzaras et al. [12], Santavicca and coworkers [13-15], Trautwein et al. [16], Cheng et al. [17], and references cited therein. The main distinction between the present measurements and these studies is a greater emphasis on the evolution of measured flame surface statistical properties during propagation from the point of ignition and the absence of preferential diffusion effects. Additionally, it is hoped that the small rates of flame stretch in comparison to extinction conditions, the simple and well-characterized turbulence field, and the information on flame surface development will be useful for developing and evaluating models of the process.

Past attempts to develop models or simulations of premixed turbulent flames in the thin laminar flamelet regime recently have been reviewed by Peters [18] and Pope [19]. Present methods are most closely related to direct numerical simula- 
tions (DNS) of turbulent premixed flames, see Ghoniem et al. [20, 21] and Ashurst and Barr [22] for early examples of this methodology. The advantage of DNS is that they provide a complete description of the process with potential to treat complications such as preferential diffusion in a fundamental way. However, DNS of both the flow field and flame propagation is computationally intensive so that treating practical flames in this manner is unlikely for some time to come [19]. Thus, the present investigation sought an approximate method that is more computationally tractable, involving a flame propagation algorithm coupled with statistical time series methods to numerically simulate velocities of the unburned gas along the flame surface, analogous to methods recently developed to treat turbulent dispersion [23] and turbulence-radiation interactions [24]. The approximate simulations were evaluated using the new measurements.

The paper begins with descriptions of experimental methods and the turbulence properties of the unburned gas. Numerical simulation of the flames is then discussed. The paper concludes with discussion of measured flame surface statistics and their comparison with numerical simulation predictions. The following discussion is brief; more details and a complete tabulation of data can be found in Kwon [25].

\section{EXPERIMENTAL METHODS}

\section{Apparatus}

The fan-stirred combustion chamber was developed by Groff [11], based on an original concept of Semenov [26]. A similar arrangement has been used by Abdel-Gayed and coworkers $[9,10]$. The chamber is quasi-spherical with a volume of 10600 $\mathrm{mL}$ and a $260-\mathrm{mm}$ cross-sectional diameter at the center. Optical access is provided by two 92 mm-diameter quartz windows in the end walls and two $10-\mathrm{mm}$-diameter windows in the side walls, with each pair of windows mounted opposite one another. The isotropic turbulent field was generated by four fans located at $90^{\circ}$ intervals along the periphery of the chamber. The fans had eight blades with a $30^{\circ}$ pitch, outer and inner diameters of 135 and $20 \mathrm{~mm}$, and a streamwise length of $23 \mathrm{~mm}$. The fans directed their flow toward the walls of the chamber and were driven by variable-speed synchronous motors. Fansler and Groff [27] show that this arrangement provides an isotropic flow field in the central region of the chamber.

Hydrogen and nitrogen (99.95\% purity) were supplied from commercial cylinders while dry air (dew point less than $240 \mathrm{~K}$ ) was obtained from laboratory supplies. The proper partial pressures of hydrogen, nitrogen and air were mixed together with the fans prior to a test. The mixture was spark ignited at the center of the chamber using electrodes extending from the top and bottom. The spark gap was roughly $3 \mathrm{~mm}$ while the sparks had a duration of $0.5 \mathrm{~ms}$ and stored energies of $0.3 \mathrm{~mJ}$. After a test, the chamber was purged with warm dry air to remove condensed water.

\section{Instrumentation}

Laser Velocimetry. Measurements involved laser velocimetry (LV) to characterize the flow properties of the unburned gas, and flame tomography (FT) for flame surface statistics. Small ( $<1 \mu \mathrm{m}$ diameter) kerosene drops were added to the gas for both techniques using a TSI 9306 atomizer. Based on observations of laminar premixed flames [25], the oil drops disappeared at the flame surface, within available resolution, similar to earlier findings of Boyer et al. [28].

Single and two-point $\mathrm{LV}$ arrangements were used. The LVs used the 514.5-nm line of a $2-\mathrm{W}$ argon-ion laser with dual-beam arrangements: 50 $\mathrm{mm}$ initial spacing $\times 250 \mathrm{~mm}$ focal length for single-point measurements and $9 \mathrm{~mm}$ initial spacing and $1000 \mathrm{~mm}$ focal length for two-point measurements. The single-point measurements involved directing the laser beams through one of the large windows, and observing the probe volume in the forward-scattering direction through the other large window, yielding a measuring volume having a diameter of $250 \mu \mathrm{m}$ and a length of $1.5 \mathrm{~mm}$. The two-point measurements involved directing the beams through one of the small windows with the small beam angle creating a probe volume that was $70 \mathrm{~mm}$ long. This probe volume was observed normal to the optical axis through the large windows with two traversable detectors, yielding measuring volumes having diameters of $200 \mu \mathrm{m}$ and lengths of $1 \mathrm{~mm}$. In both cases, rotating the beams provided 
velocity data in the vertical and horizontal directions. The laser beams were frequency shifted to eliminate directional bias and ambiguity, because turbulence intensities were generally greater than $500 \%$. Integral length scales were large $(12.5$ $\mathrm{mm}$ ) and seeding was heavy for present test conditions so that data rates were greater than 10 $\mathrm{kHz}$ using the single burst made of the burst counter signal processor, while integral time scales generally were greater than $5 \mathrm{~ms}$. Thus, the burst counter analog output was low-pass filtered and digitally sampled at equal time intervals $(10 \mathrm{kHz}$ sampling rate over a sampling time of 8s) to yield unbiased time-averaged results. Experimental uncertainties (95\% confidence) were largely limited by finite sampling times and are estimated to be less than $10 \%$ for rms velocity fluctuations and $15 \%$ for spatial correlation coefficients and temporal spectra. All measurements were repeatable within these limits over the period of testing.

Flame Tomography. A pulsed dye laser, providing $0.6 \mathrm{~J}$ of light per pulse at $514.5 \mathrm{~nm}$ and a 2- $\mu$ s pulse duration, was used for the FT measurements. The laser beam was focused with spherical and cylindrical lenses and directed through one of the small windows to produce a $200-\mu$ m-thick laser light sheet across the midplane of the combustion chamber. Light scattered from the particles in the unburned gas was recorded by a $35-\mathrm{mm}$ SLR camera (Kodak Tri-X film) viewing the light sheet normal to the optical axis through one of the large windows. A $10-\mathrm{nm}$ bandwidth laser line filter between the window and the camera reduced background radiation from the flame. The development of the flames was observed using various delays between the time of ignition and the time of the laser pulse.

The flame surface was found by tracing the edge of the region scattering light using a Gould FD 5000 Image Display. Analysis of eight realizations, for each delay time and reactant mixture ratio, yielded the mean flame radius and perimeter based on the centroid and cross-sectional area of the flame image, the rms fluctuation from the mean, the fractal dimension $\left(D_{3}\right)$ defined according to Mandelbrot [29], and the perimeter of the actual flame surface. Gouldin [30] defines outer and inner scales based on flame surface fractal properties; unfortunately, outer scales were com- parable to $\tilde{r}_{f}$ and were not very informative while inner scales could not be resolved due to the spatial resolution limits of the laser sheet $(200$ $\mu \mathrm{m})$. The experimental uncertainties of these measurements will be considered when they are discussed.

\section{Test Conditions}

The laminar flame properties of the reactant mixtures are summarized in Table 1 as a function of the volumetric fraction of oxygen in the nonfuel gas. All tests were carried out at $\phi=1.8$, which places them at the maximum laminar flame speed condition $[6,7]$. The initial pressure was $3 \mathrm{~atm}$.: pressure measurements using a piezoelectric transducer showed that combustion chamber pressure essentially remained at this value for the period when measurements were made. The density ratio, $\rho_{u} / \rho_{b}$, was found, assuming thermodynamic equilibrium in the burned gas using the Gordon and McBride [31] algorithm. The unburned gas kinematic viscosity and thermal conductivity were found using the methods of Brokaw, and Mason and Saxena described in Ref. [32], with pure gas properties drawn from Keenan et al. [33]. Laminar burning velocities were measured from schlieren motion pictures of the flame ball under quiescent conditions [1]: present measurements at 3 atm agreed with results summarized by Lewis and von Elbe [6] at 1 atm within experimental uncertainties $(10 \%)$. Characteristic laminar flame thicknesses, $\delta_{L}=\alpha / S_{L}$, are small, $1.8-3.3 \mu \mathrm{m}$, because of the relatively high laminar flame speeds and pressures of the tests.

Test conditions for the turbulent flame experiments are summarized in Table 2. Measurements

TABLE 1

Summary of Laminar Flame Properties ${ }^{a}$

\begin{tabular}{cccccc}
\hline $\begin{array}{c}\mathrm{O}_{2} /\left(\mathrm{N}_{2}+\mathrm{O}_{2}\right)^{b} \\
(-)\end{array}$ & $\begin{array}{c}\rho_{u} / \rho_{b} \\
(-)\end{array}$ & $\begin{array}{c}\alpha \\
\left(\mathrm{mm}^{2} / \mathrm{s}\right)\end{array}$ & $\begin{array}{c}\nu \\
\left(\mathrm{mm}^{2} / \mathrm{s}\right)\end{array}$ & $\begin{array}{c}S_{L}{ }^{c} \\
(\mathrm{~m} / \mathrm{s})\end{array}$ & $\begin{array}{c}\delta_{L} \\
(\mu \mathrm{m})\end{array}$ \\
\hline 0.210 & 6.4 & 4.6 & 7.6 & 2.5 & 1.8 \\
0.150 & 5.4 & 4.2 & 6.9 & 1.5 & 2.8 \\
0.125 & 5.2 & 4.0 & 6.5 & 1.2 & 3.3 \\
\hline
\end{tabular}

${ }^{a}$ Hydrogen, air, and nitrogen mixtures with a fuel-equivalence ratio of 1.8 , initial pressure of $3 \mathrm{~atm}$, and temperature of $298 \pm 3 \mathrm{~K}$.

${ }^{b}$ Relative oxygen concentration by volume.

${ }^{c}$ From Lewis and von Elbe [6]. 
TABLE 2

Summary of Turbulent Flame Test Conditions ${ }^{a}$

\begin{tabular}{ccccccc}
\hline $\begin{array}{c}\mathrm{O}_{2} /\left(\mathrm{N}_{2}+\mathrm{O}_{2}\right) \\
(-)\end{array}$ & $\begin{array}{c}N \\
(\mathrm{rpm})\end{array}$ & $\begin{array}{c}\bar{u}^{\prime} \\
(\mathrm{m} / \mathrm{s})\end{array}$ & $\begin{array}{c}\bar{u}^{\prime} / S_{L} \\
(-)\end{array}$ & $\begin{array}{c}\mathrm{Re}_{r} \\
(-)\end{array}$ & $\begin{array}{c}l_{K} \\
(\mu \mathrm{m})\end{array}$ & $\begin{array}{c}l_{K} / \delta_{L} \\
(-)\end{array}$ \\
\hline 0.210 & 1000 & 1.2 & 0.48 & 1965 & 42 & 21 \\
& 2000 & 2.4 & 0.96 & 3930 & 25 & 12 \\
0.150 & 1000 & 1.2 & 0.80 & 2095 & 40 & 13 \\
& 2000 & 2.4 & 1.60 & 4195 & 24 & 8 \\
0.125 & 1000 & 1.2 & 1.00 & 2320 & 37 & 12 \\
\hline
\end{tabular}

${ }^{a}$ Unreacted mixture properties from Table 1 ; integral length scale of turbulence of $12.5 \mathrm{~mm} ; \bar{u} / \bar{u}^{\prime}$ less than $10 \%$ for all conditions within $\pm 30 \mathrm{~mm}$ from the center of the chamber.

of turbulence properties appearing in Table 2 are discussed subsequently. A range of mixtures and rotational speeds were considered to provide $\bar{u}^{\prime} / S_{L}$ in the range $0-1.6$ and turbulence Reynolds numbers in the range $0-4195$. Kolmogorov length scales were estimated as $l_{K}=\Lambda / \operatorname{Re}_{T}{ }^{3 / 4}$ from Tennekes and Lumley [34]: they are generally an order of magnitude larger than the flame thickness, which is representative of the thin laminar flamelet regime. Mean velocities were variable over the region within $30 \mathrm{~mm}$ of the center of the chamber; however, $\bar{u} / \bar{u}^{\prime}$ was generally less than $10 \%$.

\section{Unburned Gas Properties}

Turbulence Properties. Velocity statistics of the unburned gas were measured to provide parameters needed to numerically simulate the velocity field. Fansler and Groff [27] also measured turbulence properties in this combustion chamber and their results will be compared with present findings wherever possible.

Root mean squared velocity fluctuations in the vertical and horizontal directions, $\bar{u}^{\prime}$ and $\bar{v}^{\prime}$, are plotted as a function of fan speed in Fig. 1. Results were obtained at the center as well as $\pm 30 \mathrm{~mm}$ from the center of the chamber at pressures of 1 and $3 \mathrm{~atm}$. A correlation for $\bar{u}^{\prime}$ at the center of the chamber for pressures of 1,3 , and $5 \mathrm{~atm}$, reported by Fansler and Groff [27], is also shown on the plot. The two sets of measurements are in good agreement, yielding a nearly linear increase of $\bar{u}^{\prime}$ with fan speed and relatively small effects of pressure. The value of $\bar{u}^{\prime}$ varied less than $10 \%$ over the region $\pm 30 \mathrm{~mm}$ from the center of the chamber, which also agrees with

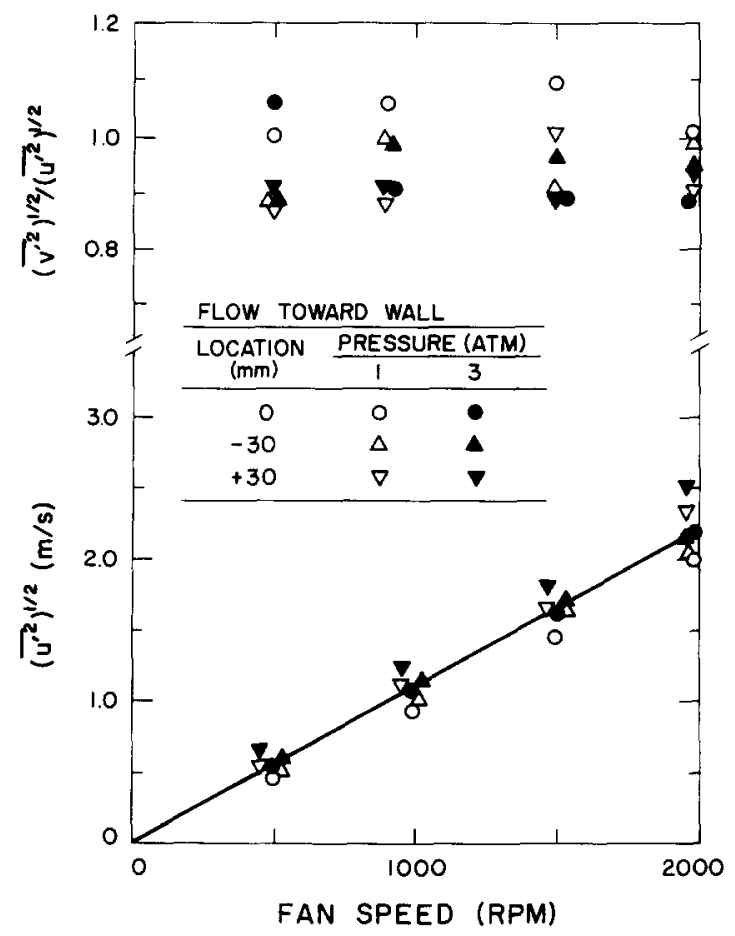

Fig. 1. Orthogonal velocity fluctuations in unburned gas.

[27]. Finally, $\bar{v}^{\prime} / \bar{u}^{\prime}$ was generally within $10 \%$ of unity over the same region, indicating reasonably isotropic turbulence. Probability density functions of velocity fluctuations, $u^{\prime}$ and $v^{\prime}$, also satisfied Gaussian probability density functions within experimental uncertainties $[25,27]$.

Temporal power spectral densities of vertical fluctuations are plotted as a function of frequency in Fig. 2. Results for various positions, fanspeeds, pressures, and velocity components are essentially the same [25]. The present integral time scales are defined by Hinze [35] as the ordinate intercepts of the power spectra divided by $4 \bar{u}^{\prime 2}$; therefore, the normalized spectra have an ordinate intercept of 4.0. Due to the high $\operatorname{Re}_{T}$, the spectra exhibit an extended inertial range where they decay proportional to the $-5 / 3$ power of frequency. The LV measuring volume was generally an order of magnitude larger than the Kolmogorov scales so that the higher rate of decay as these scales are approached could not be observed. Effects of step noise due to the finite time between LV bursts [36] are not seen in the results illustrated in Fig. 2 because data rates were more than an order of magnitude faster than the maximum frequencies of the measurements. 


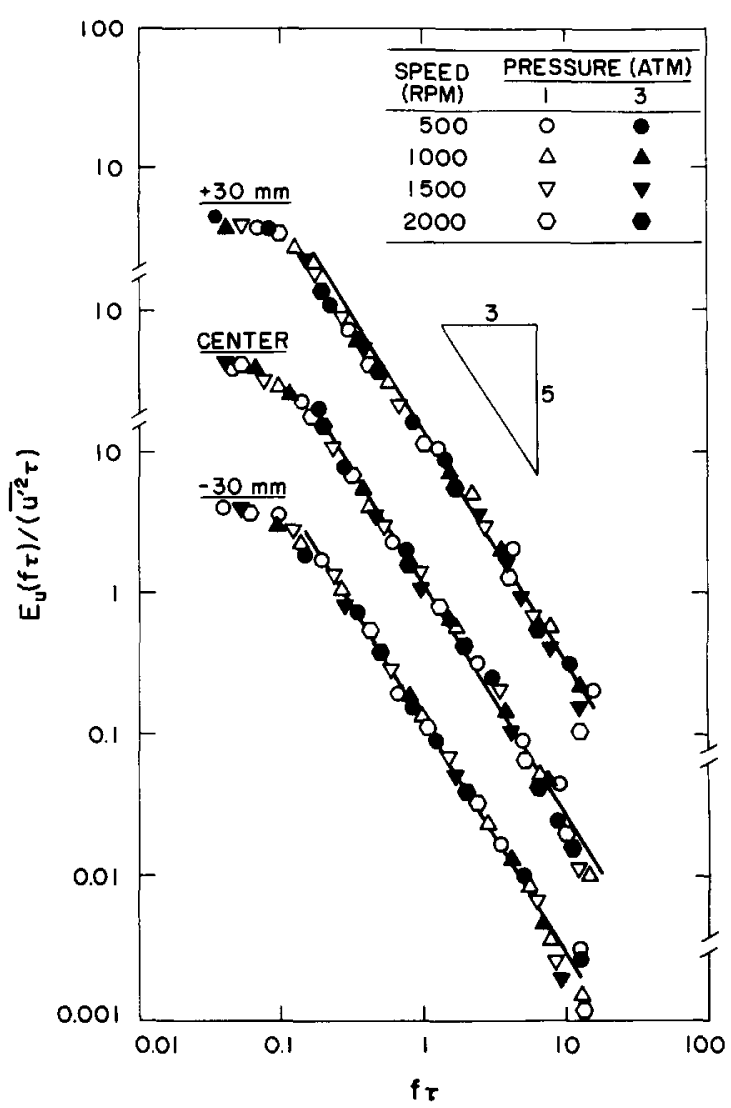

Fig. 2. Temporal power spectral densities of velocity fluctuations in unburned gas.

Present measurements of temporal integral scales are plotted as a function of fan speed in Fig. 3. Finding $\tau$ using the Hinze [35] procedure introduces uncertainties from scatter of the spectra at low frequencies, see Fig. 2; nevertheless, experimental uncertainties (95\% confidence) of $\tau$ are less than $20 \%$. A correlation of present measurements, and an earlier correlation of Fansler and Groff [27], also are shown on the plot. Effects of pressure and position on $\tau$ are not large in comparison to experimental uncertainties. The correlations follow from the assumption of nearly constant spatial integral scales, velocity fluctuations proportional to fan speeds, and temporal and spatial scales related to characteristic times on the order of integral scale sized eddies $(\tau \sim$ $\left.\Lambda / \bar{u}^{\prime}\right)$. This yields a reasonably successful correlation, $\tau(s)=12 / N(\mathrm{rpm})$ for the present measurements. Values of $\tau$ from Ref. 27 are roughly twice as large as present measurements, which is surprising because the same apparatus was used.

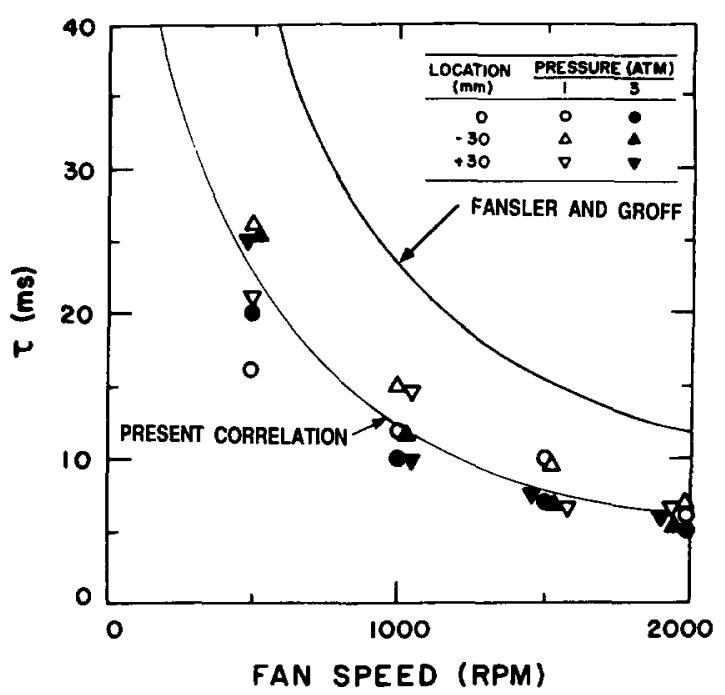

Fig. 3. Temporal integral scales in unburned gas.

The factor of 2 difference may have resulted from errors introduced due to the presence of mean velocities when recurrence rate correlations, used in Ref. 27, are corrected for directional bias. In any event, present values of $\tau$ represent conventional evaluations of $\tau$ from an experimental time series of velocity fluctuations; therefore, they were adopted for the numerical simulations.

Measurements of the transverse spatial correlation coefficients are plotted as a function of distance between two points, $\Delta x$, in Fig. 4 . Let $x$ be the coordinate axis passing through the two

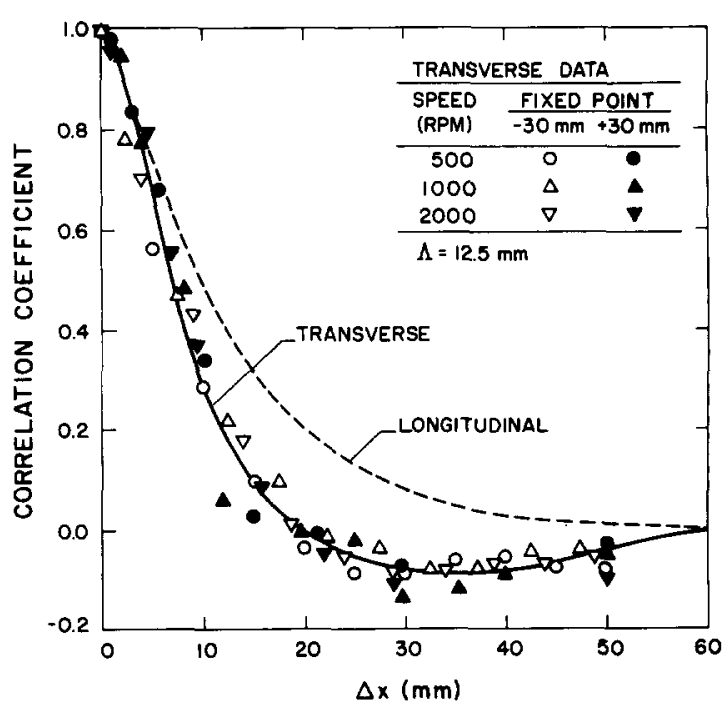

Fig. 4. Transverse and longitudinal spatial correlation coefficients in unburned gas. 
points, with $u^{\prime}$ and $v^{\prime}$ being velocity fluctuations along and normal to the $x$ axis. Then, the transverse correlation coefficient is defined as $g(\Delta x)$ $=\overline{v^{\prime}(x) v^{\prime}(x+\Delta x)} / \overline{v^{\prime 2}}$. This correlation is relatively independent of fan speed and position within the central region of the chamber. Conservation of mass in isentropic turbulence requires a region where $g(\Delta x)<0$, yielding a shape somewhat like a Frenkiel function [35].

The longitudinal spatial correlation, $f(\Delta x)$ $=\overline{u^{\prime}(x) u^{\prime}(x+\Delta x)} / \overline{u^{\prime 2}}$ also is required for numerical simulation of the velocity field. It was not possible to measure $f(\Delta x)$ using the present LV configuration; therefore, it was computed from the fit of the present measurements of $g(\Delta x)$ through the differential equation relating the two for isotropic turbulence [35]:

$d / d \Delta x\left(\Delta x^{2} f(\Delta x)\right)=2 \Delta x g(\Delta x)$,

where $f(0)=1$ by definition. The resulting plot of $f(\Delta x)$ is also illustrated in Fig. 4. Due to the small values of the Kolmogorov length scales, the curvature of $f(\Delta x)$ could not be resolved at small $\Delta x$, yielding roughly an exponential shape over the range of the present measurements. Integrating $f(\Delta x)$ yielded the spatial integral scale, $\Lambda=12.5 \mathrm{~mm}$. This length scale is roughly half the value found by Fansler and Groff [27] from their recurrence-rate temporal correlation measurements, similar to the differences in temporal integral scales between the two studies that were discussed earlier.

Mean Velocities. Mean radial velocities in the unburned gas were measured as the flames propagated from the center of the chamber. These measurements employed the single-channel LV with the measuring volume located $30 \mathrm{~mm}$ from the center of the chamber. The velocities for various values of $\bar{r}_{f}$ were ensemble averaged over eight tests to yield an experimental uncertainty ( $95 \%$ confidence) less than $15 \%$ for mean velocities greater than $10 \%$ of the maximum mean velocity.

Measured values of $\bar{u}$ at a fixed radial location during flame propagation were compared with predictions assuming that the flame surface represents a volumetric source in the mean, due to the density change at the surface, and inviscid spherically symmetric constant density mean flow of the reactant gas. This yields

$\bar{u} /\left(S_{T}\left(\rho_{u} / \rho_{b}-1\right)\right)=\left(\bar{r}_{f} / r\right)^{2}$

Measured values of $\bar{u}$ are plotted as suggested by Eq. 2 in Fig. 5 . The density ratios used in the normalization appear in Table 1 while $S_{T}$ and $\bar{r}_{f}$ were found from the FT measurements to be discussed later. Except for conditions far from the flames, where low velocities limit measuring accuracy, Eq. 2 provides an excellent correlation of the data. This implies that the chamber walls did not exert a significant effect on flow properties in the region where measurements were made. Equation 2 also provides a simple description of mean velocities in the unburned gas for the numerical simulation.

\section{Theoretical Methods}

General Description. Turbulent flame propagation was numerically simulated using the flame propagation algorithm of Chorin [37] coupled with statistical time series simulation [4] of the velocity field in the unburned gas along the flame surface. The flame propagation algorithm was adapted from MIMOC [38] and only can provide a two-dimensional time-dependent simulation. This a major limitation, but it was desired to evaluate the simplified simulation before extending it to treat three-dimensional effects.

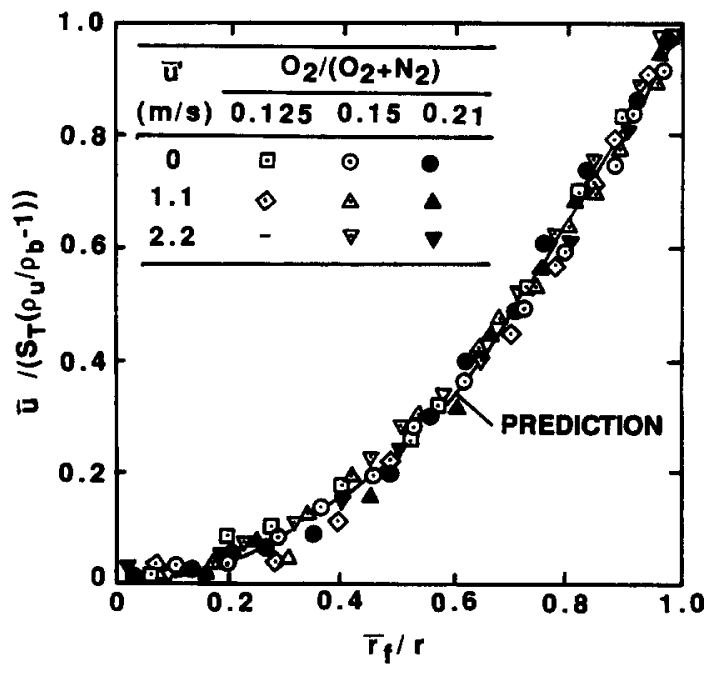

Fig. 5. Mean velocities in the unburned gas during flame propagation. 
Other major assumptions of the numerical simulation are as follows: constant pressure turbulent deflagration wave; infinitely thin flame sheet with constant unburned and burned gas properties; stationary homogeneous isotropic turbulence in the unburned gas, unaffected by the presence of the flame; neutrally stable flame with negligible effects of quenching, that is, relative to the gas, the flame propagates normal to its surface at the laminar burning velocity; and mean velocity field in unburned gas found assuming that the flame acts like a spherically-symmetric volumetric source (Eq. 2).

The constant pressure and thin flamelet assumptions are conditions of the experiments: the chamber pressure rise was negligible in the region where measurements were made while characteristic flame thicknesses (see Table 1) were 10-100 times smaller than Kolmogorov length scales and available spatial resolution. The assumption that turbulence properties of the unburned gas are not affected by the flame is an open issue for the present test flames. However, Videto and Santavicca [13] do not observe significant changes of unburned gas turbulence properties for free premixed turbulent flames. This implies that the volumetric expansion of the flame passively convects the unburned gas turbulence field away from the ignition source. Neutral stability is a condition of the experiments while effects of quenching are not large because present flames were at the maximum laminar burning velocity condition, well away from extinction conditions, and had modest values of $\bar{u}^{\prime} / S_{L}$. Finally, the volumetric source approximation is justified by the measurements illustrated in Fig. 5.

Statistical simulations of the velocity field can be designed to satisfy any number of the statistical properties of turbulence: mean velocities, the pdf of velocity fluctuations, temporal and spatial correlations, cross correlations, instantaneous conservation of mass, higher-order correlations, etc. Priorities must be set, however, because computation and memory requirements increase as the number of properties to be simulated increase. Thus, present calculations were limited to simulating mean velocities, Gaussian pdfs of velocity fluctuations, and temporal and spatial correlations. Cross autocorrelations were ignored because they are zero for isotropic turbulence.
Satisfying conservation of mass has not been important for other statistical simulations of turbulence $[23,24]$ and is partly accounted for by the properties of the longitudinal and transverse spatial correlations discussed in connection with Eq. 1. Considering higher-order correlations is not very attractive because this information is rarely available.

A final approximation was to represent the spatial and temporal correlations as exponential functions because this yields a Markov-like simulation that substantially simplifies the computations [4, 23, 24]. Exponential fits are reasonably good for temporal and longitudinal spatial correlations but do not represent the negative portion of the Frenkiel function shape of the transverse spatial correlation (Fig. 4). Nevertheless, the approximation was adopted for the transverse spatial correlation as well, because the significant portion of the correlation curve, where the values of the correlation are near unity, is reasonably represented by an exponential function.

Velocity Simulation. Mean velocities are known from Eq. 2, because the simulation provides a running estimate of $\bar{r}_{f}$ and $S_{T}$ (taken as averages over the flame perimeter at each instant); therefore, only velocity fluctuations must be simulated. The flame mostly affects the calculations through the mean velocity; therefore, the turbulent velocity field of the unburned gas was found for the whole flow (to minimize bookkeeping problems) even though only the portion near the flame surface was needed for the flame propagation algorithm. This inefficient approach was acceptable because the velocity simulation required much less computer time than the flame propagation algorithm.

An autoregressive process was used for the simulation; therefore, the velocity fluctuation at the point to be computed was a weighted sum of velocity fluctuations computed earlier and a random shock [4]. Each component of velocity at a point can be found independently because they are statistically independent for isotropic turbulence. The process will be formulated to find the velocity fluctuations at a generic point $x_{0}, y_{0}, t_{0}$ in the flow field, assuming that previous computations have found velocity fluctuations at $n$ previous points, for example, $x_{1}, y_{1}, t_{1} ; \ldots ; x_{n}$, $y_{n}, t_{n}$. This numerical ordering of previous points 
can be arranged in any convenient manner because only correlations among points must be considered explicitly, not their positions in space and time. With this arrangement, the unknown velocity fluctuations at 0 are found from the following autoregressive processes [4]:

$u_{o}^{\prime}=\sum_{i=1}^{p} U_{o i} u_{i}^{\prime}+s_{u 0} ; \quad v_{o}^{\prime}=\sum_{i=1}^{p} V_{o i} v_{i}^{\prime}+s_{\mathrm{v} 0}$

where $p \leq n$ is selected to eliminate points having small correlation coefficients with respect to point 0 . The $U_{0 i}, V_{0 i}$ are weighting factors selected so that the simulated correlations between points can be satisfied. The $s_{u 0}$ and $s_{v 0}$ are uncorrelated random variables (random shocks) having Gaussian pdfs with mean values of zero and variances selected to match the pdfs of $u_{0}^{\prime}$ and $v_{0}^{\prime}$.

To procedure to find the properties of the weighting factors and random shocks is identical to any other autoregressive process. Taking $u_{0}^{\prime}$ as an example, the $U_{0 i}$ can be found by solving the following system of linear algebraic equations, given the correlations between points $[4,24]$ :

$\overline{u_{0}^{\prime} u_{k}^{\prime}}=\sum_{i=1}^{p} U_{o i} \overline{u_{i}^{\prime} u_{k}^{\prime}} ; \quad k=1, \ldots, p$

Once the $U_{o i}$ are determined, the variance of $s_{u 0}$ can be found from

$\bar{s}_{u 0}^{2} / \bar{u}_{0}^{\prime 2}=1-\sum_{i=1}^{p} U_{o i}\left(\overline{u_{1}^{\prime} u_{0}^{\prime}} / \bar{u}_{0}^{\prime 2}\right)$.

This provides all properties needed to find $u_{0}^{\prime}$, and analogously $v_{0}^{\prime}$, from Eqs. 3 for any point 0 . Finally, $u_{0}=\bar{u}_{0}+u_{0}^{\prime}$, where $\bar{u}_{0}$ is found from Eq. 2 given $\bar{r}_{f}$ and $S_{T}$ from the flame propagation algorithm.

As a practical matter, resolution of the flame surface in space and time requires relatively small spatial and temporal increments in comparison to the integral scales; therefore, the correlation coefficients of the important nearest neighbors of the point being computed are all near unity. Then it is reasonable to decompose general correlations into products of correlations along the individual coordinate axes as follows:

$$
\begin{gathered}
\overline{u^{\prime}(x, y, t) u^{\prime}(x-i \Delta x, y-j \Delta y, t-k \Delta t)} \\
\bar{u}^{\prime 2}=F^{i} G^{j} T^{k}
\end{gathered}
$$

where

$$
\begin{aligned}
& F=\exp \left(-\Delta x / \Lambda_{f}\right), \quad G=\exp \left(-\Delta y / \Lambda_{g}\right) \\
& T=\exp (-\Delta t / \tau)
\end{aligned}
$$

are the correlation coefficients for single increments in each coordinate direction. Equation 6 is formally correct in each coordinate direction, for example, $i$ varying with $j=k=0$, due to the properties of exponential functions. However, it is only approximate for general variations of $i, j$, and $k$. In particular, for $\Delta x=\Delta y$, $\overline{u^{\prime}(x, y, t) u^{\prime}(x-\Delta x, y-\Delta y, t)} / \bar{u}^{\prime 2}=(F$ $\left.\sqrt{2}+G^{\sqrt{2}}\right) / 2$ rather than FG, etc. [35]. Nevertheless, the difference between the two expressions is less than $10 \%$ for $F$ and $\mathrm{G}$ ca. 0.9 so that the error is acceptable in view of the other approximations of the simulation. Finally, the mean motion of the gas affects the spatial and temporal correlations with respect to observations from a fixed Eulerian grid. This effect was considered, however, $\Delta \tau / \tau$ and $\bar{u} \Delta \tau / \Lambda \ll 1$, etc., for present conditions so that the correction was small. Thus, $F, G$, and $T$, as well as $\bar{u}^{\prime}$ and $\bar{v}^{\prime}$, were essentially constant over the flow field for the present conditions.

Under the approximations of the previous paragraph, Kwon [25] shows that only the seven nearest neighbors of the point being computed have values of $U_{0 i}$ and $V_{0 i}$ that are not zero. This behavior is similar to pure time series simulations with exponential temporal correlations (Markov processes) where an autoregressive process including only the previous time step still satisfies the temporal correlation for all time [4].

The locations of the seven points of the approximate simulations, and the corresponding weighting factors and variances of the random shocks, are summarized in Table 3 . Initial conditions at $t=0$, and boundary points at $x=0$ or $y=0$, require corrected procedures because all seven points are not available. In these cases, the unavailable points can simply be deleted while dropping the corresponding terms in the expressions for $\bar{s}_{u 0}{ }^{2}$ and $\bar{s}_{v 0}{ }^{2}$ (note that the corresponding 
TABLE 3

Parameters of the Approximate Simulation ${ }^{a}$

\begin{tabular}{|c|c|c|c|}
\hline$i$ & Location & $U_{o i}$ & $V_{o i}$ \\
\hline 1 & $x-\Delta x, y, t$ & F & G \\
\hline 2 & $x, y-\Delta y, t$ & $G$ & $\mathrm{~F}$ \\
\hline 3 & $x, y, t-\Delta t$ & $\mathrm{~T}$ & $T$ \\
\hline 4 & $x-\Delta x, y-\Delta y, t$ & $-F G$ & $-F G$ \\
\hline 5 & $x-\Delta x, y, t-\Delta t$ & $-\mathrm{FT}$ & $-\mathrm{GT}$ \\
\hline 6 & $x, y-\Delta y, t-\Delta t$ & $-\mathrm{GT}$ & $-\mathrm{FT}$ \\
\hline 7 & $x-\Delta x, y-\Delta y, t-\Delta t$ & FGT & FGT \\
\hline \multicolumn{4}{|c|}{$\begin{aligned} \bar{s}_{u 0}^{2} / \bar{u}^{\prime 2}= & \overline{s_{u 0}} / \bar{v}^{\prime 2}=1-F^{2}-G^{2}-T^{2} \\
& +(F G)^{2}+(F T)^{2}+(G T)^{2}-( \\
F= & \exp \left(-\Delta x / \Lambda_{f}\right), G=\exp \left(-\Delta x / \Lambda_{g}\right) \\
T= & \exp (-\Delta t / \tau)\end{aligned}$} \\
\hline
\end{tabular}

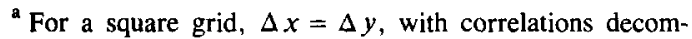
posable as products according to Eq. 6 and for stationary homogeneous and isotropic turbulence.

terms are simply $U_{0 i}{ }^{2}$ or $V_{0 i}^{2}$ ). For example, $u_{0}^{\prime}$ at $x=y=t=0$ implies that no previous points are available so that $u_{0}^{\prime}=s_{u 0}$ and $\overline{s_{u 0}{ }^{2}}=\bar{u}^{\prime 2}$.

The approximate simulation was tested for grid arrangements and values of $F, G$, and $T$ typical of actual simulations. Plots of typical simulated temporal and spatial correlations are illustrated in Fig. 6. This simulation involved $F=T=0.95$ for $100 \times 100$ spatial grid and 4000 time steps. The temporal correlation is shown for a point near the center of the grid while spatial correlations in the vertical and horizontal directions provide two realizations of this property. The correlations are reproduced reasonably well in the region where they are large. Larger errors and irregular behavior of the spatial correlations at large separation distances are due to sampling limitations, and can be reduced by averaging over more realizations [25]. Probability density functions of velocity fluctuations were also reproduced quite well over the grid [25].

\section{Flame Propagation Computations}

Flame surface properties were found using the flame propagation portion of MIMOC [38]. This involves the simple line interface calculation (SLIC) of Noh and Woodward [39] to treat advection of the flame surface at $u=\bar{u}+u^{\prime}$ and $v=\bar{v}+v^{\prime}$, and the implementation of Huygens' principle by Chorin [37] to propagate the flame

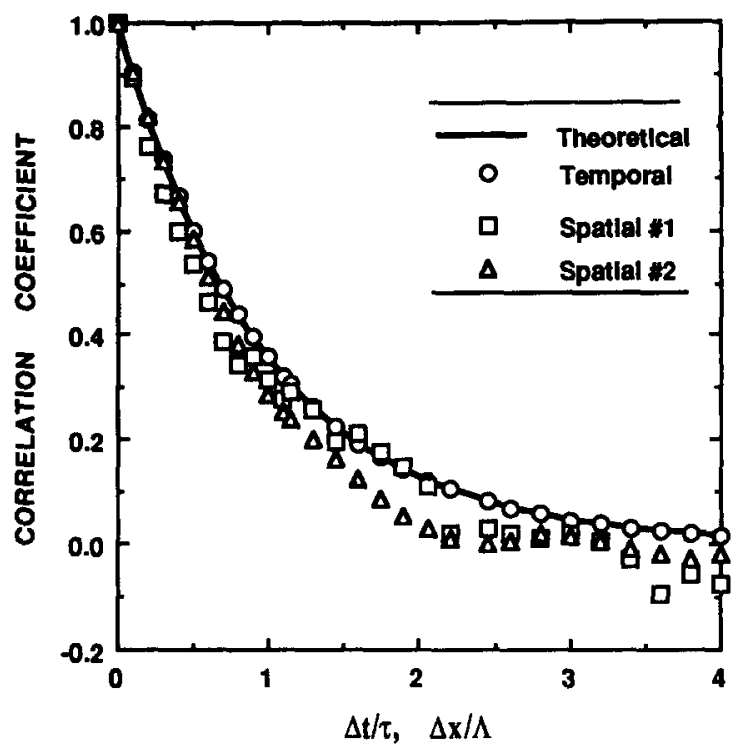

Fig. 6. Simulations of spatial and temporal correlations.

relative to these velocities at its laminar burning velocity and normal to its surface. The modification of the original algorithm [38] by Sethian [40], to remove bias of off-axis propagation caused by the order of $x$ and $y$ sweeps, was adopted for the present calculations.

Results reported here are based on a $100 \times 100$ grid with $\Delta x=\Delta y=1 \mathrm{~mm}$ and $\Delta t=$ $0.03-0.08 \mathrm{~ms}$. Numerical accuracy was evaluated by finding $\bar{r}_{f}, \bar{r}_{f}^{\prime}, D_{3}$, and $P_{T} / P_{L}$ for grid sizes $1 / 2,1$, and 2 times as large as the final computations. Extrapolation of the results indicated that the numerical uncertainties of the results reported here are less that $4 \%$, which is small in comparison to uncertainties introduced by the other approximations of the simulation. The same flame surface properties as the measurements were found by analyzing 16 realizations. The uncertainties of each simulated flame property will be considered when results for the property are discussed.

\section{RESULTS AND DISCUSSION}

\section{Flame Visualization}

Typical flame tomographs for the present neutrally stable conditions at the highest values of $\bar{u}^{\prime} / S_{L}$ used are illustrated in Fig. 7. The tomographs are for $\bar{r}_{f}=15,30$, and $45 \mathrm{~mm}$. The 


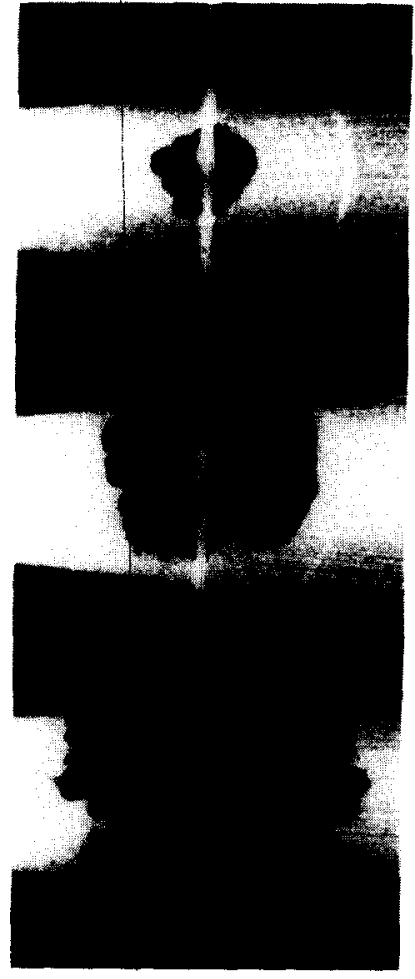

$\mathrm{u}^{\prime} / \mathrm{S}_{\mathrm{L}}=1.6$
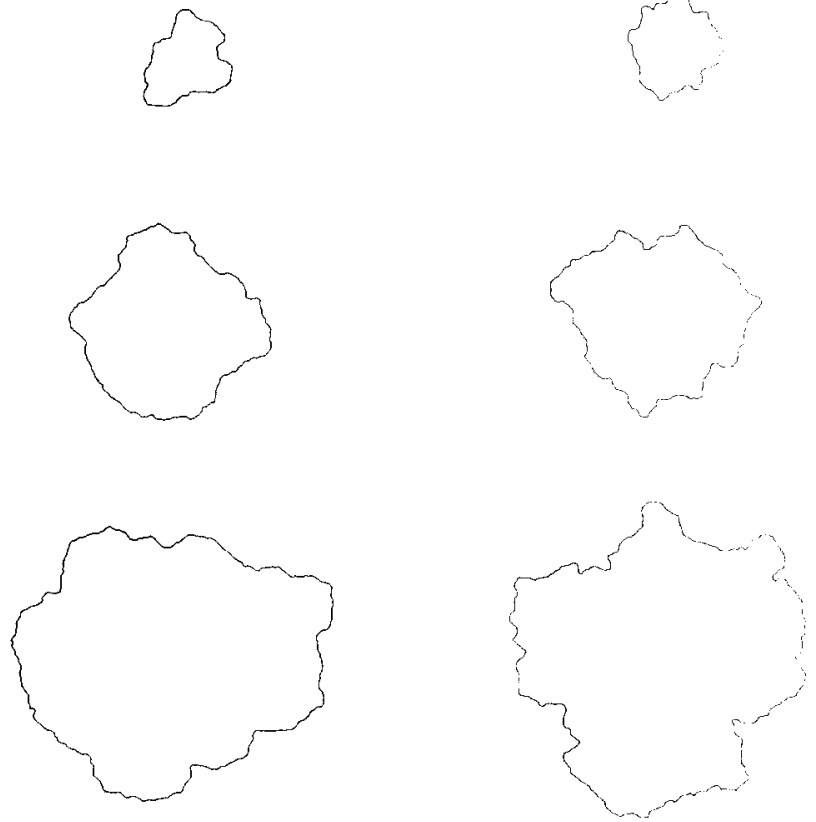

$\mathrm{u}^{\prime} / \mathrm{S}_{\mathrm{L}}=3.2$ (SIM.)

Fig. 7. Observed and simulated flame surface images: $\mathrm{O}_{2} /\left(\mathrm{N}_{2}+\mathrm{O}_{2}\right)=0.15$.

results show a progressive increase of flame surface distortion with increasing mean radius (i.e., time). This behavior is similar to the progressively increasing distortions of the flame surface of premixed turbulent jet flames with increasing distance from the flameholder [1], aside from motion of the centroid of the free flame image (which was small for present test conditions).

Simulated flame surfaces at $\bar{u}^{\prime} / S_{L}=1.6$ (the experimental value) and 3.2 are also illustrated in Fig. 7. Both simulations represent the trend that flame surface distortion increases with mean flame radius (time). The main effect of increasing $\bar{u}^{\prime} / S_{L}$ is to increase the degree of distortion of the surface at particular values of $\bar{r}_{f}$ so that the larger $\bar{u}^{\prime} / S_{L}$ yields a more irregular surface with finer-grained distortion. Both simulations are qualitatively similar to the flame tomographs, but the small-scale distortions are better represented by the results for $\bar{u} / S_{L}=3.2$ (which is twice the experimental value). More quantitative assess- ment of the simulations will be undertaken by considering flame surface statistics.

\section{Flame Surface Statistics}

Moments. The measured and simulated variations of $\bar{r}_{f}$ and $\bar{r}_{f}^{\prime}$ as a function of time after ignition are illustrated in Figs. 8-10 for all conditions tested. In order to avoid disturbances from the spark ignition process, measurements begin at $\bar{r}_{f}=10 \mathrm{~mm}$, which is reached roughly $1-2 \mathrm{~ms}$ after ignition. Trends of flame properties at earlier times are complex due to disturbances from the electrodes and the spark discharge. Thus, the measurements do not extrapolate linearly to $\bar{r}_{f}=$ 0 at $t=0$ while computational noise dominates simulation properties at small times due to limited spatial resolution. In order to eliminate these effects, measured and simulated times when $\bar{r}_{f}=$ $10 \mathrm{~mm}$ have been made coincident, although the times shown in Figs. 8-10 are times after the 


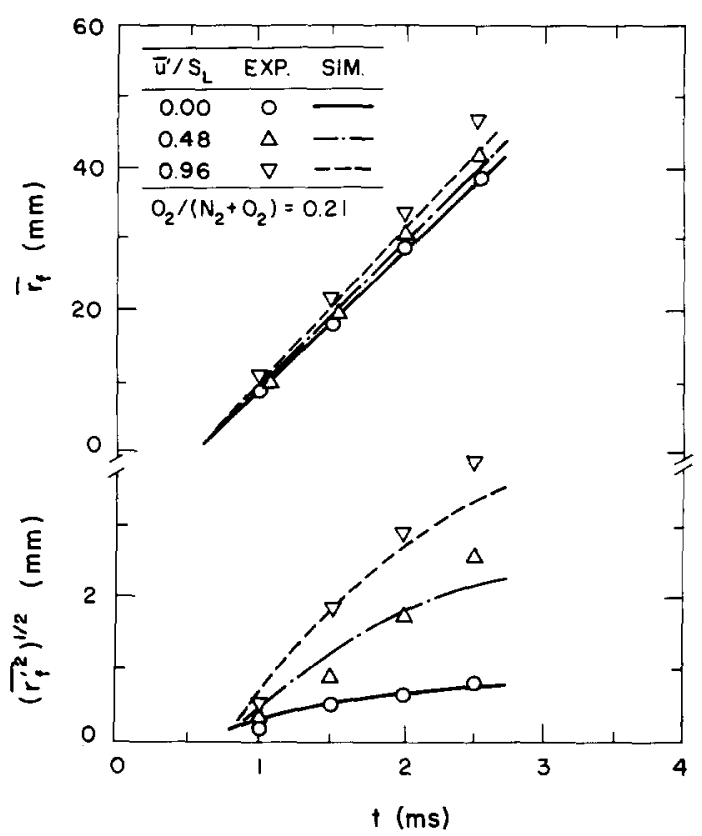

Fig. 8. Mean and fluctuating flame radius as a function of time: $\mathrm{O}_{2} /\left(\mathrm{N}_{2}+\mathrm{O}_{2}\right)=0.210$.

start of spark discharge. The uncertainties $(95 \%$ confidence) of $\bar{r}_{f}$ and $\bar{r}_{f}^{\prime}$ are estimated to be less than $13 \%$ and $15 \%$ for the measurements and $7 \%$ and $9 \%$ for the simulations, respectively, with these uncertainties dominated by the limited number of realizations.

Measured values of $\bar{r}_{f}$ increase linearly with time for laminar conditions, $\bar{u}^{\prime} / S_{L}=0$, for the time period illustrated in Figs. 8-10. This is expected because the flames are neutral with respect to preferential diffusion instability and the flame surfaces do not self-distort [8]. The linear increase of $\bar{r}_{f}$ with time is also preserved for small values of $\bar{u}^{\prime} / S_{L}$ and time, although $d \bar{r}_{f} / d t$ at a particular $\bar{r}_{f}$ progressively increases as $\bar{u}^{\prime} / S_{L}$ increases due to increased levels of flame surface distortion by the turbulence. At larger times and $\bar{u}^{\prime} / S_{L}$, however, $d \bar{r}_{f} / d t$ increases with increasing time as well, because effects of the progressive growth of flame surface distortion become large enough to be resolved.

Measured values of $\bar{r}_{f}^{\prime}$ also increase with time for the results illustrated in Figs. 8-10. Finite, but small, values of $\bar{r}_{f}^{\prime}$ are also observed for $\bar{u}^{\prime} / S_{L}=0$, even though the flame surface is smooth. This is caused by distortion of the overall flame shape due to the presence of the spark

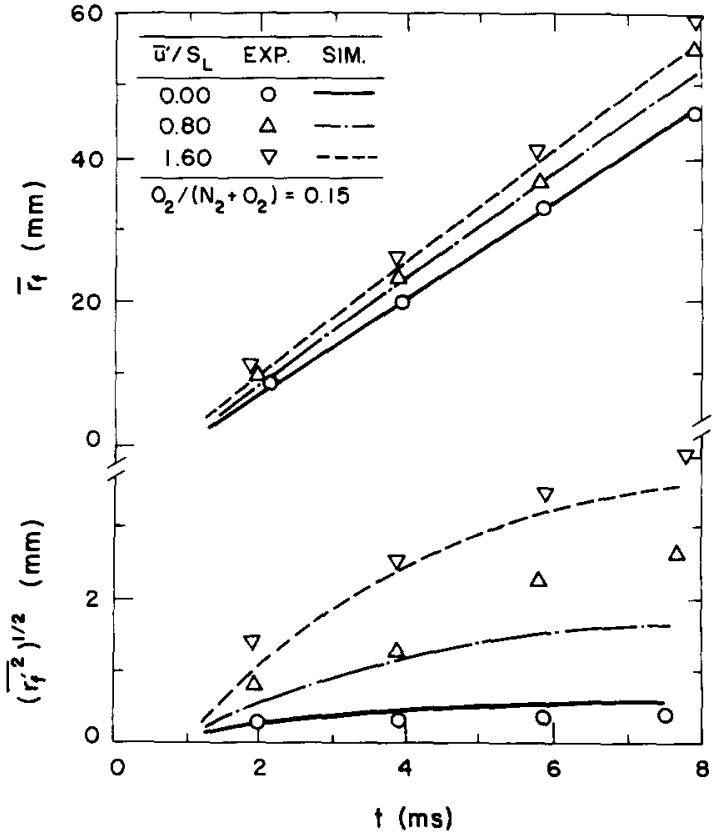

Fig. 9. Mean and fluctuating flame radius as a function of time: $\mathrm{O}_{2} /\left(\mathrm{N}_{2}+\mathrm{O}_{2}\right)=0.150$.

electrodes and small flow disturbances in the chamber. For nonzero values of $\bar{u}^{\prime} / S_{L}$, the flame kernel is initially smooth and nearly spherical. With increasing time, however, the flame surface becomes progressively more distorted by the tur-

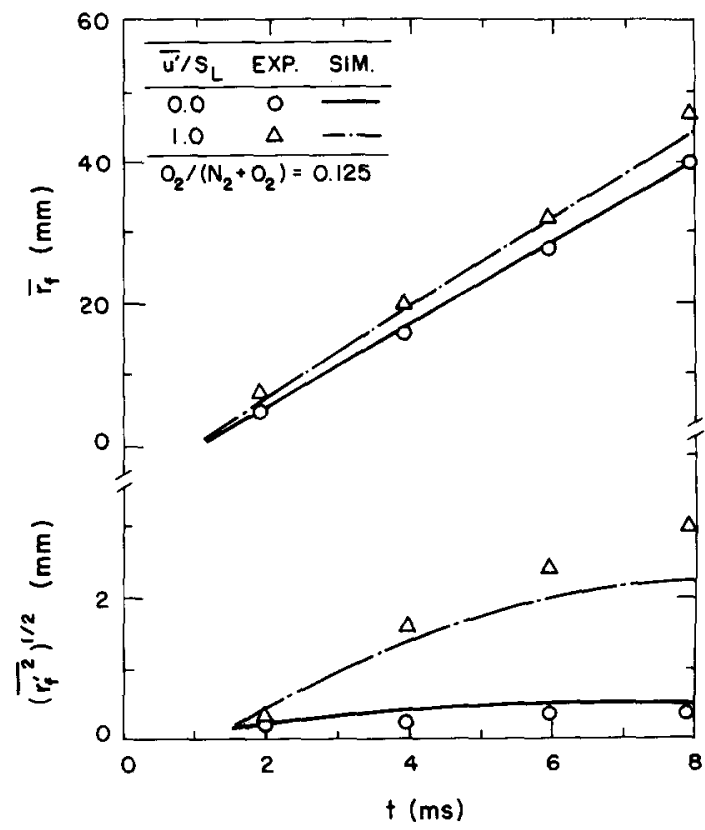

Fig. 10. Mean and fluctuating flame radius as a function of time: $\mathrm{O}_{2} /\left(\mathrm{N}_{2}+\mathrm{O}_{2}\right)=0.125$. 
bulence. The rate of increase of $\bar{r}_{f}^{\prime}$ with time increases as $\bar{u}^{\prime} / S_{L}$ increases because larger velocity fluctuations imply larger deformations of the surface in a given time interval. Although $\bar{r}_{f}^{\prime}$ increases significantly in the time period illustrated in Figs. 8-10, maximum values are generally less than $30 \%$ of the spatial integral scale $(12.5 \mathrm{~mm})$. The reason for this is that turbulent deformation of the flame surface does not have much time to develop due to the relatively large turbulent flame speed for present test conditions, for example, maximum times of propagation are in the range 0.4-1.3 integral time scales.

Naturally, the simulated $\bar{r}_{f}$ at $\bar{u}^{\prime} / S_{L}=0$ agree with measurements in Figs. 8-10 because surface distortion and limitations of a two-dimensional simulation are absent for laminar conditions. Effects of $\bar{u}^{\prime} / S_{L}$ on the variation of $\bar{r}_{f}$ with time are also predicted reasonably well by the simulations when these effects are small. There are greater deficiencies, however, in the region where $d \bar{r}_{f} / d t$ itself begins to noticeably increase with time (large $\bar{u}^{\prime} / S_{L}$ and long times) where the simulations underestimate the rate of increase of $d \bar{r}_{f} / d t$. This is felt to be due primarily to the limitations of a two-dimensional simulation of a three-dimensional turbulent process. In particular, a two-dimensional time-dependent simulation treats the flame like a ruled surface in the third dimension, suppressing irregularities of the flame surface caused by out of plane fluctuations. Although this is thought to be the main source of the underestimation of $d \bar{r}_{f} / d t$, and other measures of flame surface distortion to be discussed subsequently, other approximations of the simulation may be factors as well, for example, effects of the flame on unburned gas turbulence properties, the limited number of turbulence properties simulated, the exponential approximations of the correlations, etc.

Predictions of $\bar{r}_{f}^{\prime}$ for $\bar{u}^{\prime} / S_{L}=0$ are illustrated in Figs. 8-10 in order to quantify numerical distortions introduced by the flame propagation algorithm. In particular, while $\bar{r}_{f}^{\prime}$ should be zero for $\bar{u}^{\prime} / S_{L}=0$, finite values are predicted that fortuitously agree with the measurements. These variations are caused by the limited spatial resolution of the calculations, incomplete correction of off-axis bias within the advection algorithm [40], and the limited directional resolution of the Huygens' principle implemented to treat flame propa- gation [37]. The effect of these difficulties tends to decrease as $\mathrm{O}_{2} /\left(\mathrm{N}_{2}+\mathrm{O}_{2}\right)$ decreases, but it is generally less than $1 \mathrm{~mm}$ for the time period considered, and generally less than $30 \%$ of predicted values of $\bar{r}_{f}^{\prime}$ for finite values of $\bar{u}^{\prime} / S_{L}$.

Simulated values of $\bar{r}_{f}^{\prime}$ exhibit trends similar to the measurements in Figs. 8-10; namely, $\bar{r}_{f}^{\prime}$ increases with time and the rate of increase tends to increase as $\bar{u}^{\prime} / S_{L}$ becomes larger. However, the simulations generally underestimate $\bar{r}_{f}^{\prime}$. This behavior is consistent with the corresponding underestimation of $d \bar{r}_{f} / d t$ by the simulation, as discussed earlier.

Fractal Dimensions. Recently, the fractal dimensions of turbulent premixed flame surfaces have received a great deal of attention as a means of quantifying the degree of wrinkledness [12-15, 30]. The main parameters are the fractal dimensions of the surface, $D_{3}$, and the fractal dimension of the intersection of the surface with a plane, $D_{2}$ [29]. The value of $D_{2}$ was found by measuring the flame perimeter, $L$, using rulers of different size, $\epsilon$, similar to past work [1].

Typical plots of $L$ as a function of $\epsilon$ for the simulations are illustrated in Fig. 11. These results are for $\bar{u}^{\prime} / S_{L}=1.6$ at various values of $\bar{r}_{f}$, ensemble-averaging results for each ruler size

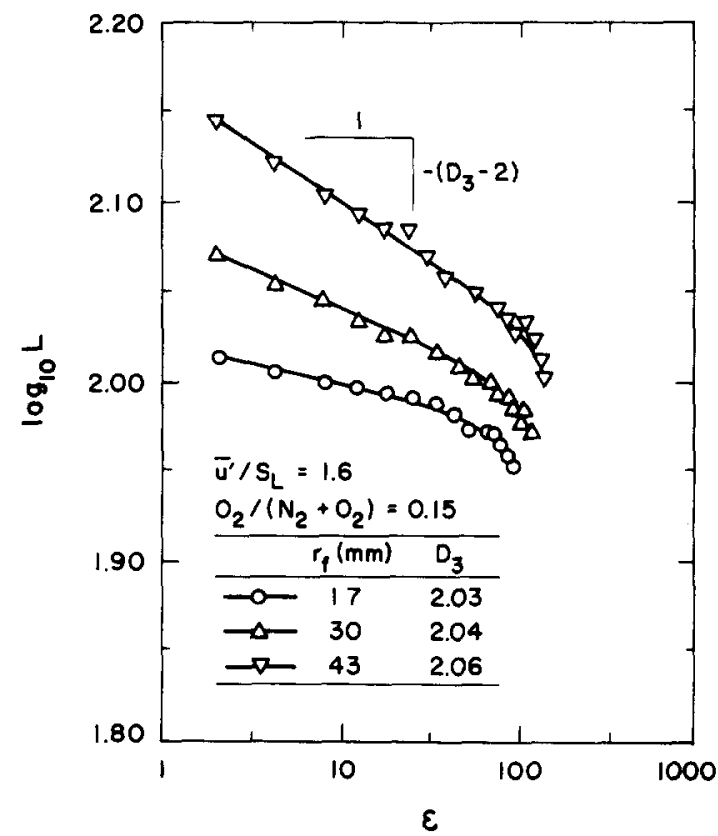

Fig. 11. Typical evaluation of the fractal dimension, $D_{3}$, for the simulation. 
over 16 realizations in order to smooth the plot. Except for regions near small and large values of $\epsilon$, where the ruler sizes approach available spatial resolution and the mean diameter of the flame, the curves exhibit a relatively smooth slope that is characteristic of fractal-like behavior. The slope of the intermediate region is $1-D_{2}$, assuming that the flame surface is fractal, and $D_{3}=D_{2}+1$, assuming that the flame surface is isotropic [29]. The value of $D_{3}$ in previous flame studies has varied between 2 , for smooth geometrical surfaces like spheres, to $2.3-2.4$, which is representative of maximum levels of distortion of constant property or premixed flame surfaces within turbulent flow fields $[12,14,41,42]$.
Measured values of fractal dimensions were found in the same manner as Fig. 11 from the flame tomographs. In this case, results for eight realizations were averaged at each test condition and $\bar{r}_{f}$ to obtain relatively smooth plots of $L$ as a function of $\epsilon$. Experimental uncertainties of these measurements were limited by the finite number of samples and the available span of $\epsilon$ to find the slope. The resulting uncertainties $(95 \%$ confidence) of $D_{3}-2$ are estimated to be less than $20 \%$. Evaluation of $D_{3}$ from the simulations yielded similar uncertainties.

Measured and simulated values of $D_{3}$ are plotted as a function of $\bar{r}_{f}$ in Fig. 12. Measurements are illustrated for all test conditions, grouped

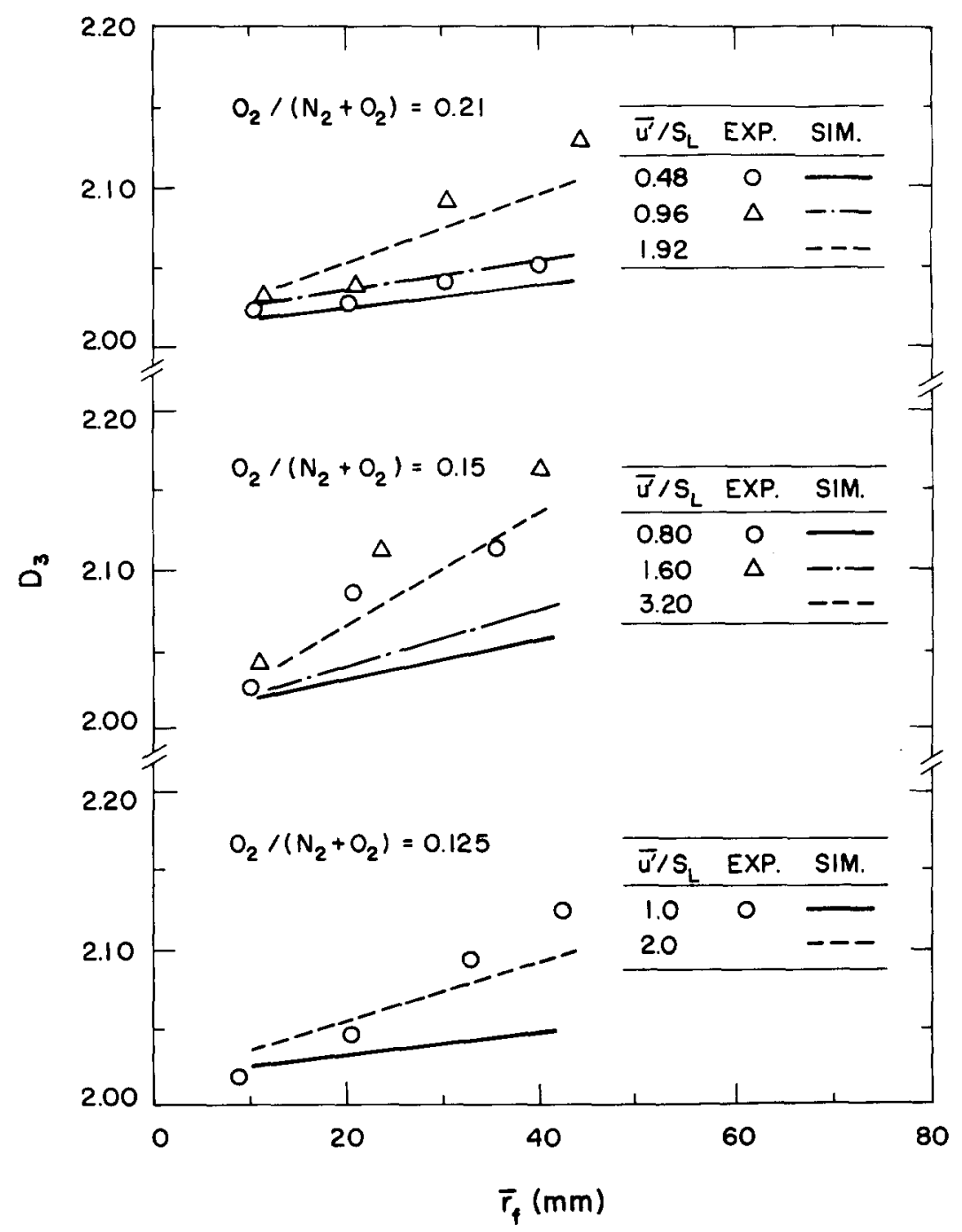

Fig. 12. Fractal dimension, $D_{3}$, as a function of flame radius. 
according to $\mathrm{O}_{2} /\left(\mathrm{N}_{2}+\mathrm{O}_{2}\right)$. Simulations of $\mathrm{D}_{3}$ are illustrated for parametric values of $\bar{u}^{\prime} / S_{L}$. At small $\bar{r}_{f}, D_{3}$ is nearly 2.0 , which is representative of the smooth spherical spark kernel. The subsequent increase is due to progressive deformation of the flame surface by turbulence, somewhat analogous to the behavior of $D_{3}$ for turbulent jet flames with increasing distance from the flame holder [1]. Maximum values of $D_{3}$, however, are not large, generally less than 2.15 . This is caused by the limited propagation time of present tests, which is 0.4-1.3 times the integral time scale as noted earlier. In terms of distance, these measurements only correspond to distances of propagation into the unreacted gas mixture on the order of one spatial integral scale (the larger displacements of $\bar{r}_{f}$ in Fig. 12 are caused by volumetric expansion of the flames due to their density ratio, see Table 1). Based on results observed at largest distances from the flame holder for premixed turbulent jet flames [1], it is expected that $D_{3}$ would eventually approach values in the range 2.3-2.4 that are characteristic of passive constant property surfaces in isotropic turbulent fields. However, measurements for larger values of $\bar{r}_{f} / \Lambda$ are needed to confirm this behavior.

Simulated values of $D_{3}$ in Fig. 12 also exhibit a progressive increase with increasing flame radius. However, use of the experimental value of $\bar{u}^{\prime} / S_{L}$ yields simulated values of $D_{3}$ significantly below the measurements for each value of $\bar{r}_{f}$. Analogous to the individual realizations illustrated in Fig. 7, however, doubling $\bar{u}^{\prime} / S_{L}$ for the simulation yields a reasonably good estimate of the variation of $D_{3}$ with $\bar{r}_{f}$. A probable reason for this deficiency is that out of plane distortions of the flame surface, which should contribute to its irregularity, are suppressed by two-dimensional simulations.

Flame Perimeter. Another measure of the distortion of the flame surfaces by turbulence was found by measuring the perimeters of flame surfaces. For present conditions flame wrinkles are not large and it is reasonable to assume that flame surface properties are isotropic, that is, flame surface statistics found for orthogonal planes through the flame centroid should be the same, so that the average surface area of the wrinkled surface can be related to the perimeter as follows
[1]:

$A_{T} / A_{L}=2\left(P_{T} / P_{L}\right)-1$,

where $P_{L}$ and $A_{L}$ are the perimeter and area of the mean flame surface at each instant. Additionally, for neutral preferential diffusion conditions and modest stretch rates, $A_{T} / A_{L}$ is a measure of $S_{T} / S_{L}$ [30].

Measured values of $P_{T} / P_{L}$ were found from eight realizations with uncertainties $(95 \%$ confidence) of $P_{T} / P_{L}-1$ estimated to be less than $25 \%$, assuming that the flame surface was fully resolved. Results for the simulations involved averages over 16 realizations, yielding uncertainties of $P_{T} / P_{L}-1$ of less than $15 \%$. The contribution of scales smaller than the resolution of the measurements $(200 \mu \mathrm{m})$ can be estimated from present measurements of $D_{3}$ through the fractal properties of the surface $[1,30]$. Conservatively estimating the minimum scale of flame wrinkling as the Kolmogorov scale and $D_{3}=2.15$ (which was the maximum value observed), unresolved distortions could contribute up to $40 \%$ increase of $P_{T}$ from values reported here. However, present values of $D_{3}$ generally were smaller than 2.15 , other estimates of the minimum scale of flame wrinkling (like the Gibson scale [18]) are larger than the Kolmogorov scale, and the resolution of the measurements and simulations were similar, so that this effect should not have a large impact on present considerations.

Measured values of $P_{T} / P_{L}$ for all the test conditions are plotted as a function of $\bar{r}_{f}$ in Fig. 13, grouped according to $\mathrm{O}_{2} /\left(\mathrm{N}_{2}+\mathrm{O}_{2}\right)$. Values of $P_{T} / P_{L}$ from the simulations also are shown on the plots, for parametric values of $\bar{u}^{\prime} / S_{L}$ that match the experiments, as well as twice the maximum experimental values, as before. The measurements show that $P_{T} / P_{L}$ is near unity for small values of $\bar{r}_{f}$, which is representative of the nearly smooth spark kernel in this region. Similar to $D_{3}$, however, $P_{T} / P_{L}$ progressively increases with increasing $\bar{r}_{f}$, with the rate of increase being more rapid as $\bar{u}^{\prime} / S_{L}$ increases. No tendency for $P_{T} / P_{L}$ to approach a limit at larger $\bar{r}_{f}$ is observed for the present relatively short propagation times. Associating $P_{T} / P_{L}$ with $A_{T} / A_{L}$ through Eq. 18, and with $S_{T} / S_{L}$ through the considerations of Gouldin [30], indicates that $S_{T} / S_{L}$ increases with $\bar{r}_{f}$, with $\bar{u}^{\prime} / S_{L}$ largely controlling the rate of increase. Thus, correla- 


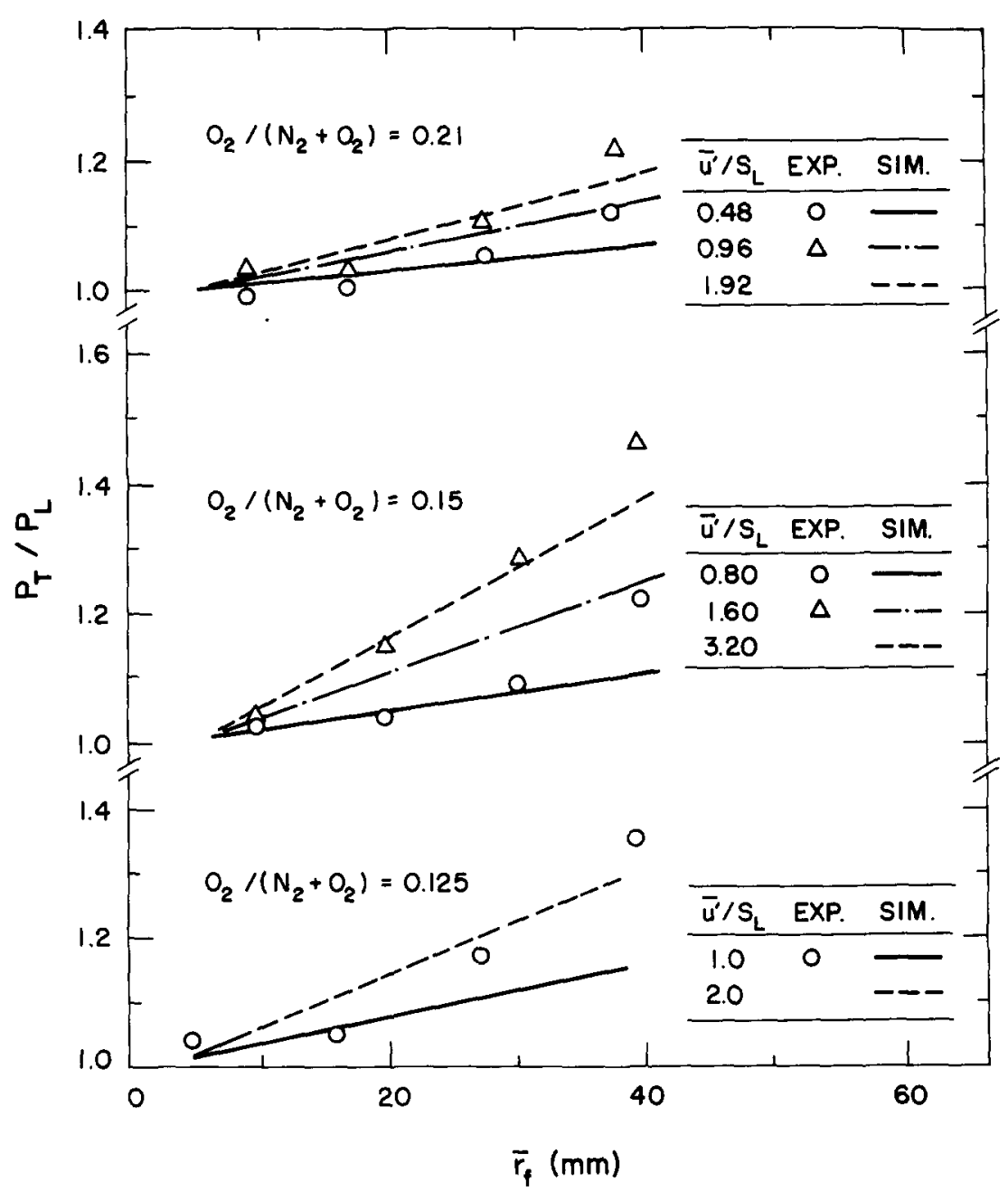

Fig. 13. Normalized turbulent flame perimeter as a function of flame radius.

tions of $S_{T} / S_{L}$ solely as a function of $\bar{u}^{\prime} / S_{L}$ are only appropriate for particular values of $\bar{r}_{f}$, as recently shown by Trautwein et al. [16] for a similar range of $\bar{r}_{f}$.

Whether $P_{T} / P_{L}$, and thus $S_{T} / S_{L}$, eventually approaches a large $\bar{r}_{f}$ limit for free turbulent flames with fixed unburned gas turbulence properties is an open issue, even though $D_{3}$ approaches the limit for constant property surfaces in turbulent environments. On one hand, if the outer fractal scale is proportional to the integral scale as proposed in [30], $S_{T}$ should eventually approach a limit at large $\bar{r}_{f}$ for these conditions. On the other hand, however, present measurements and those of Ref. 1 find outer fractal scales generally proportional to the maximum dimen- sions of the flame, which implies that $S_{T}$ should continuously increase as $\bar{r}_{f}$ increases, based on the approach of Ref. 30. Clearly, tests of free turbulent flames at longer times in comparison to the integral time scale are needed so that this important property of turbulent flames can be resolved.

The simulations in Fig. 13 yield the correct trends of $P_{T} / P_{L}$ with increasing $\bar{r}_{f}$ and $\bar{u}^{\prime} / S_{L}$, with use of values of $\bar{u}^{\prime} / S_{L}$ that are twice the experimental values yielding best agreement with the measurements as before. Thus, the simulation appears to be promising, but more definitive evaluation of its performance will require consideration of a three-dimensional time-dependent version of the simulation. 


\section{CONCLUSIONS}

The major conclusions of the study can be summarized as follows:

1. Flame tomograph measurements showed that $d \bar{r}_{f} / d t, \bar{r}_{f}^{\prime}, \quad D_{3}$, and $P_{T} / P_{L}$ (and thus $S_{T} / S_{L}$ ) increase with time (distance) from the point of ignition. Observed times and distances of propagation, however, were on the order of integral scales in the unreacted gas so that results represent a developing period of flame distortion by the turbulence. At larger times and distances, $D_{3}$ is expected to approach values found for constant property surfaces in isotropic turbulence and the variation of flame properties with time (distance) should change: measurements are needed to resolve behavior in this limiting region.

2. Measurements showed that increasing $\bar{u}^{\prime} / S_{L}$ tends to increase the rate of turbulent distortion of the flame surface with time (distance) from the point of ignition. Thus, previous correlations of $S_{T} / S_{L}$ and $D_{3}$ solely as a function of $\bar{u}^{\prime} / S_{L}$ were found because they reflect this rate of increase for limited ranges of flame dimensions. Nevertheless, such correlations are incomplete unless they account for the development of flame surface properties. Models or correlations of turbulent premixed flame properties that only consider local conditions, and do not account for effects of time (distance) from the point of ignition, are intrinsically incomplete. A possible exception is behavior at long times when $D_{3}$ may approach values for constant property surfaces in turbulence but this still must be studied.

3. The present numerical simulation successfully predicted the trends of flame surface properties but underestimated their rate of increase with time (distance) from the point of ignition. Reasonably accurate predictions could be obtained by the artifice of doubling $\bar{u}^{\prime} / S_{L}$ in order to compensate for a two-dimensional simulation, however, the general effectiveness of this approach is unknown. Extension to three-dimensional simulations is computationally feasible, even for high Reynolds number flames, and should be undertaken to evaluate the methodology more thoroughly. Other aspects of the simulation that need to be assessed include the following: Are unburned gas turbulence properties significantly modified as the flame surface is approached? Is simulation of moments, pdfs and spatial and temporal correlations in the unburned gas sufficient to treat flame surface distortion? What are the limitations of the approach as $\delta_{L}$ becomes comparable to $l_{K}$, and characteristic flame development times approach turbulent time scales? Can effects of preferential diffusion and quenching be successfully simulated?

This research was supported by the Office of Naval Research, contract No. N00014-87-K0698 with S. Ramberg serving as Scientific Program Officer. The authors also wish to thank General Motors Research Laboratories for donation of the combustion chamber and E. G. Groff and T. Fansler for useful discussions concerning the research.

\section{REFERENCES}

1. Wu, M. S., Kwon, S., Driscoll, J. F., and Faeth, G. M., Combust. Sci. Technol. 78:69-96 (1991).

2. Bray, K. N. C., in Turbulent Reacting Flows (P. A. Libby and F. A. Williams, Eds.), Springer-Verlag, Berlin, 1980, pp. 115-135

3. Abraham, J., Williams, F. A., and Bracco, F. V., SAE Paper No. 850345, 1985.

4. Box, G. E. P., and Jenkins, G. M., Time Series Analysis, Holden Day, San Francisco, 1976, pp. 47-66

5. Manton, J., von Elbe, G., and Lewis, B., J. Chem. Phys. 20:153-158 (1952).

6. Lewis, B., and von Elbe, G., Combustion Flames and Explosions of Gases, Academic, New York, 1961, pp. 381-384.

7. Andrews, G. E., and Bradley, D., Combust. Flame 20:77-89 (1973).

8. Wu, M. S., Kwon, S., Driscoll, J. F., and Faeth, G. M., Combust. Sci. Technol. 73:327-350 (1990).

9. Abdel-Gayed, R. G., and Bradley, D., Sixteenth Symposium (International) on Combustion, The Combustion Institute, Pittsburgh, 1976, pp. 1725-1735.

10. Abdel-Gayed, R. G., Bradley, D. Hamid, M. N., and Laws, M., Twentieth Symposium (International) on Combustion, The Combustion Institute, Pittsburgh, 1984, pp. 505-512.

11. Groff, E. G., Combust. Flame 67:153-162 (1987).

12. Mantzaras, J., Felton, P. G., and Bracco, F. V., Combust. Flame 77:295-310 (1989).

13. Videto, B. D., and Santavicca, D. A., Combust. Sci. Technol. 70:47-73 (1990).

14. Santavicca, D. A., Liou, D., and North, G. L., SAE Paper No. 900024, 1990. 
15. North, G. L., and Santavicca, D. A., Combust. Sci. Technol. 72:215-232 (1990).

16. Trautwein, S. E., Grudno, A., and Adomeit, G. Twenty-Third Symposium (International) on Combustion, The Combustion Institute, Pittsburgh, 1990 , pp. 723-728.

17. Cheng, W. K., Keck, J. C., Hainsworth, E., Lai, M. C., Ferreira, E., Hurlburt, E., Pope, S. B., and Girimaji, S. S., Turbulent Premixed Flame Study, Final Report, Contract DE-FG02-86ER 13553, MIT, Cambridge, MA, 1990

18. Peters, N., Twenty-First Symposium (International) on Combustion, The Combustion Institute, Pittsburgh, 1988, pp. 1231-1250.

19. Pope, S. B., Twenty-Third Symposium (International) on Combustion, The Combustion Institute, Pittsburgh, 1990, pp. 591-612.

20 Ghoniem, A. F., Chorin, A. J., and Oppenheim, A. K., Eighteenth Symposium (International) on Combustion, The Combustion Institute, Pittsburgh, 1980, pp. 1375-1383.

21. Ghoniem, A. F., Chorin, A. J., and Oppenheim, A. K., Trans. R. Soc. Lond. A394:303-315 (1982).

22. Ashurst, W. T., and Barr, P. K., Combust. Sci. Technol. 34:227-256 (1983).

23. Parthasarathy, R. N., and Faeth, G. M., J. Fluid Mech. 220:515-537 (1990).

24. Kounalakis, M. E., Sivathanu, Y. R, and Faeth, G. M., J. Heat Transf, 113:437-445 (1991).

25. Kwon, S., Premixed Hydrogen/Air Flames in Isotropic Turbulence, Ph.D. thesis, University of Michigan, Ann Arbor, 1990.

26. Semenov, E. S., Combust. Expl. Shock Waves 1:57-62 (1965).

27. Fansler, T. D., and Groff, E. G., Combust. Flame 80:350-354 (1990)
28. Boyer, L., Clavin, P., and Sabathier, F., Eighteenth Symposium (International) on Combustion, The Combustion Institute, Pittsburgh, 1980, pp. 1041-1049.

29. Mandelbrot, B. B., J. Fluid. Mech. 72:401-416 (1975).

30. Gouldin, F. C., Combust. Flame 68:249-266 (1987).

31. Gordon, S., and McBride, G. J., Computer Program for Calculations of Complex Chemical Equilibrium Compositions, Rocket Performance, Incident and Reflected Shocks, and Chapman-Jouguet Detonations, NASA Report SP-273, Washington, 1971.

32. Reid, R. C., Prausnitz, J. M., and Sherwood, T. K., The Properties of Gases and Liquids, 3rd ed., McGraw-Hill, New York, 1977, pp. 391-516.

33. Keenan, J. H., Chao, J., and Kaye, J., Gas Tables, Wiley, New York, 1980.

34. Tennekes, H., and Lumley, J. L., Turbulence, M.I.T. Press, Cambridge, MA, 1972, pp. 67.

35. Hinze, J. O., Turbulence, 2nd ed., McGraw-Hill, New York, 1975, Chap. 3.

36. Adrian, R. J., and Yao, C. S., Exp. Fluids 5:17-28 (1987).

37. Chorin, A. J., J. Comp. Phys. 35:1-11 (1980).

38. Ghoniem, A. F., Marek, C. J., and Oppenheim, A. K., Modeling Interface Motion of Combustion (MIMOC), NASA TP-2132, 1983.

39. Noh, W. T., and Woodward, P., Fifth International Conference on Numerical Methods in Fluid Dynamics (A. I. Vooren and P. J. Zandbergen, Eds.), Springer-Verlag, Berlin, 1976, pp. 330-339.

40. Sethian, J., J. Comp. Phys. 54:425-456 (1984).

41. Lovejoy, S., Science 216:185-187 (1982).

42. Sreenivasan, K. R., and Meneveau, C., J. Fluid Mech. 173:357-386 (1986).

Received 2 January 1991; revised 29 September 1991 